\title{
HEMINEFRECTOMÍA LAPAROSCÓPICA EN ECTOPIA RENAL CRUZADA CON FUSION INFERIOR DE UNIDADES RENALES
}

\author{
Octavio A. Castillol,2, Rafael Sánchez-Salas', Alejandro Foneron' y Gonzalo Vitagliano'.
}

Departamento de Urología'. Clínica Indisa. Departamento de Urología². Facultad de Medicina. Universidad de Andres Bello. Chile.

Resumen.- OBJETIVO: La ectopia renal cruzada es una anomalía congénita infrecuente. Presentamos el caso de un niño de 3 años de edad con una ectopia renal cruzada derecha, con fusión de unidades renales y con historia previa de infecciones urinarias recurrentes y procedimientos quirúrgicos fallidos.

MÉTODOS: Paciente de 3 años con diagnóstico de ectopia renal cruzada y fusión inferior de unidades renales se sometió a una heminefrectomía laparoscópica de la unidad renal inferior, producto de una hidronefrosis severa de la unidad inferior e infecciones urinarias a repetición:

\section{CORRESPONDENCIA}

Octavio Castillo

Department of Urology

Clínica Indisa

Avenida Santa María 1810

7530234 Providencia

Santiago de Chile. Chile.

octaviocastillo@vtr.net

Aceptado para publicar: 3 de enero 2009
RESULTADOS: Se realizo una heminefrectomía laparoscópica de la unidad renal inferior sin incidentes. El tiempo operatorio fue de 200 minutos El postoperatorio transcurrió sin incidentes. El paciente fue dado de alta a las 18 horas post cirugía. Después de un seguimiento de 5 años el paciente permanece asintomático, sin infecciones urinarias y con buena función de la unidad renal remanente.

CONCLUSIONES: El abordaje laparoscópico es una alternativa viable para el manejo de esta patología entregando todas las ventajas de la cirugía mínimamente invasiva.

Palabras clave: Anomalía congénita. Ectopia renal cruzada con fusión. Laparoscopia. Heminefrectomía.

Summary.- OBJECTIVES: Crossed fused renal ectopia is a rare congenital anomaly. We report the case of a 3 year old boy with diagnosis of right crossed fused renal ectopia, history of recurrent urinary tract infection and previous failure of surgical treatment.

METHODS: Three year old boy with diagnosis of right crossed fused renal ectopia of the inferior moiety underwent a laparoscopic heminephrectomy of the inferior renal unit, due to severe hidronefrosis and recurrent urinary tract infections.

RESULTS: A laparoscopic right heminephrectomy of the inferior renal moiety was performed uneventfully. Operation room time was 200 minutes and there were no perioperative complications. Patient was discharged 18 hours after the procedure. After 5 years of follow up patient remains asymptomatic with good renal function.

CONCLUSIONS: The laparoscopic approach is an acceptable option to treat this anomaly, with all the advantages of minimally invasive surgery. 
Keywords: Congenital anomaly. Crossed fused renal ectopia. Laparoscopic heminephrectomy.

\section{INTRODUCCIÓN}

La ectopia renal cruzada constituye una malformación congénita infrecuente, con una incidencia que va entre $1 / 1350$ y $1 / 2000$ de los nacimientos. Esta anomalía ocurre mas comúnmente en hombres, con una relación de $2: 1$, siendo la ectopia de izquierda a derecha tres veces mas frecuente. Los riñones unilaterales fusionados con ectopia inferior son la variedad mas frecuente $(1,2)$. Los pacientes habitualmente permanecen asintomáticos hasta la $4^{\underline{a}}$ ó $5^{a}$ década, comenzando en este momento con infecciones urinarias, urolitiasis, masa o dolor abdominal (2). Hay tres patrones diferentes de fusión renal: el riñón en herradura, el riñón en herradura con fusión lateral, y la ectopia renal cruzada. Se ha descrito un patrón embriogénico que explica los modelos asimétricos de fusión, los que pueden estar dados por el efecto que ejerce la flexión lateral y rotación de la porción caudal del feto, en la posición de las cuerdas nefrogénicas y los ductos de Wolff (3). La ectopia renal cruzada se ha asociado con anomalías congénitas sincrónicas en un $50 \%$ de los casos, siendo las del tracto urinario las mas frecuentes (4). El estudio diagnostico puede ser llevado a cabo mediante una ultrasonografía abdominal, tomografía axial computada o una pielografía endovenosa (5).

\section{CASO CLÍNICO}

Paciente masculino de 3 años de edad, con cuadro compatible con una infección urinaria complicada con una pielonefritis, es enviado para evaluación. Presenta antecedente de infecciones urinarias a repetición complicadas y el diagnostico de ectopia renal cruzada con fusión renal así como hidroureteronefrosis bilateral, hecho a los cuatro meses de vida. Dentro de los antecedentes destacan además una ureterosotomía cutánea en 1998 y una ureteroneocistostomía tipo Cohen el 2000. El seguimiento posterior revelo adecuada función renal hasta tres meses después del último procedimiento.

El paciente sufrió una recaída con infecciones urinarias y la evaluación radiológica mostró una ectopia renal cruzada derecha con fusión renal con hidroureteronefrosis severa de la unidad renal inferior (Figura 1). Los parámetros de laboratorio, incluyendo la creatinina, estaban dentro de rangos normales, por lo que se indico una heminefrectomía laparoscópica.
El procedimiento se realizó con tres puertos y una óptica de 0 grados de $5 \mathrm{~mm}$. El paciente se colocó en decúbito lateral izquierdo y se realizó un neumoperitoneo a $12 \mathrm{mmHg}$ mediante técnica cerrada con aguja de Veress. El primer puerto de $5 \mathrm{~mm}$ se ubicó a nivel del ombligo. Un puerto de $12 \mathrm{~mm}$ se colocó en la región subcostal derecha, mientras que otro de $5 \mathrm{~mm}$ se colocó en la fosa iliaco derecha (Figura 2).

\section{TÉCNICA QUIRÚRGICA}

1. Liberación del hemi colon derecho.

2. Identificación y disección de uréter dilatado y de la pelvis rotada lateralmente. El uréter dilatado tenía $20 \mathrm{~mm}$ de diámetro y se continuaba a proximal con una pelvis igual de dilatada.

3. Identificación, disección y control vascular de los vasos de la unidad renal inferior. Tanto arteria como vena, se dirigían hacia los vasos ilíacos. Para su control se utilizaron clips de titanio.

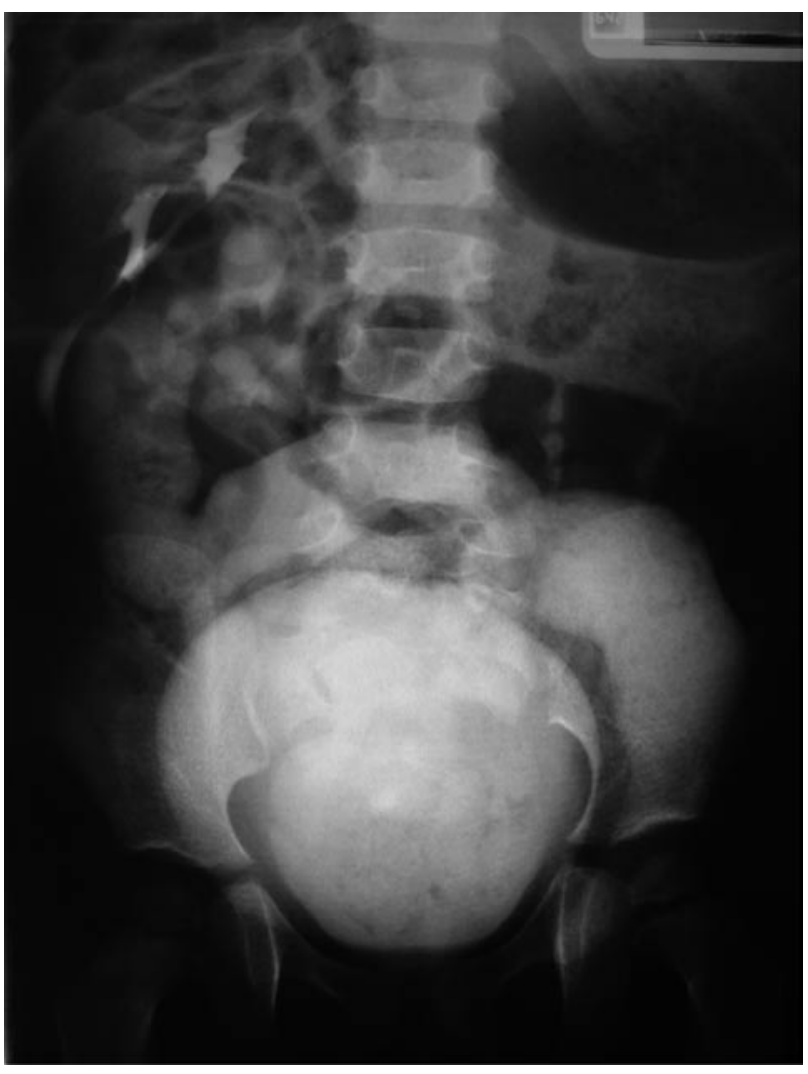

FIGURA 1. Evaluación radiológica que muestra la ectopia renal cruzada con fusión y severa hidroureteronefrosis. 


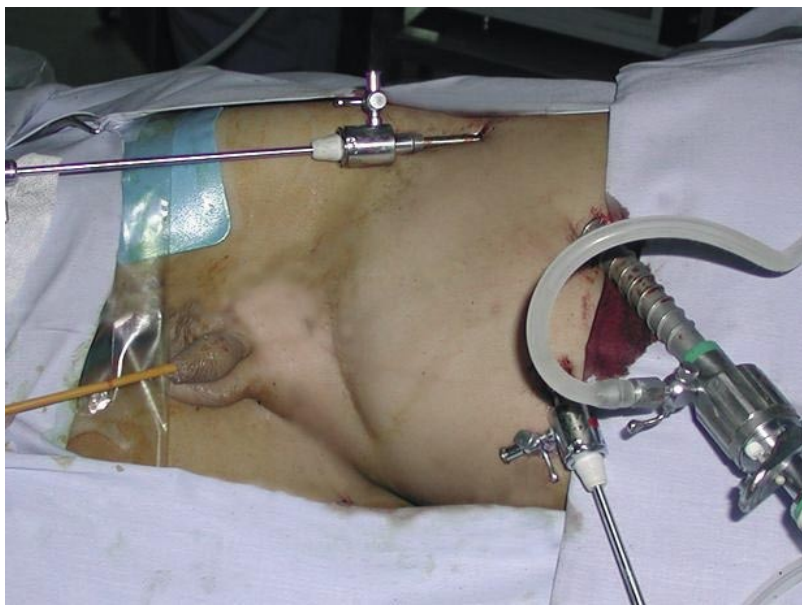

FIGURA 2. Posición del paciente y de los puertos.

4. Resección de la unidad inferior. Se identifica el límite de resección producido por isquemia y se realiza la sección del parénquima renal mediante el bisturí harmónico. (Ethicon Endosurgery).

5. Evaluación endoscópica del campo quirúrgico. Se identificó una apertura de un cáliz inferior, que se reparó por vía laparoscópica con sutura intracorpórea, utilizando poligactin 4-0.

6. La extracción de la pieza se realizó a través de una incisión de $3 \mathrm{~cm}$ sobre una incisión de Pfanennstiel previa (Figura 3).

El tiempo operatorio fue de 200 minutos con un sangrado estimado de $100 \mathrm{cc}$. El postoperatorio transcurrió sin incidentes. Los requerimientos analgésicos fueron mínimos. El régimen habitual se reinició 6 horas después del procedimiento. El paciente fue dado de alta a las 18 horas post cirugía. Después de un seguimiento de 5 años el paciente permanece asintomático, sin infecciones urinarias. Una pielografía endovenosa confirmó el buen estado de la unidad renal superior remanente (Figura 4).

\section{DISCUSIÓN}

El tratamiento de los pacientes con ectopia renal cruzada representa un desafío quirúrgico. Los avances en la laparoscopia facilitan el abordaje quirúrgico y entregan medios para realizar procedimientos complejos con óptimos resultados (6). Reportes previos han demostrado el éxito del tratamiento laparoscópico de la ectopia renal $(6,7)$.

Stanley et al. (7) reportaron una heminefrectomía laparoscópica en una unidad renal superior de

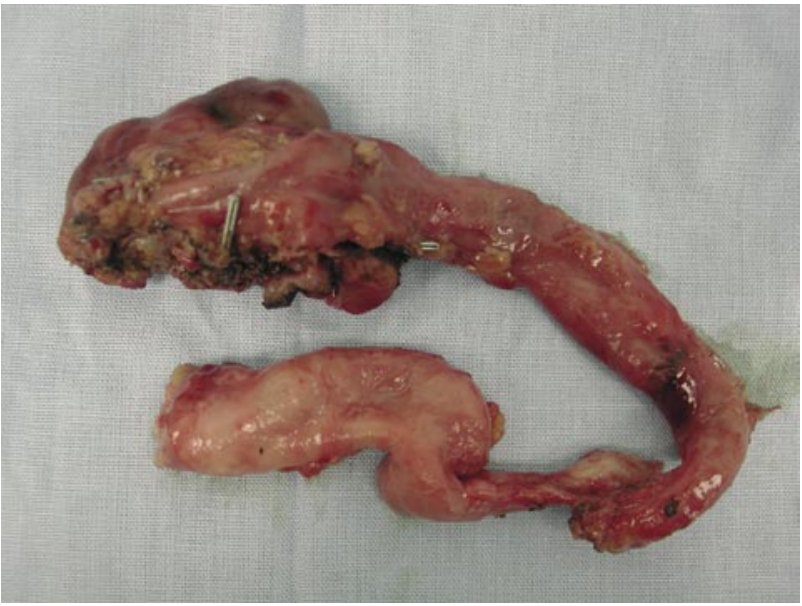

FIGURA 3. Pieza quirúrgica.

una ectopia renal cruzada con resultados adecuados en un paciente de 18 años. Pietrow et al. (6) describieron le primera resección laparoscópica mano asistida en ectopia renal cruzada con una enfermedad poliquística severa. Andersen et al. (8) descri-

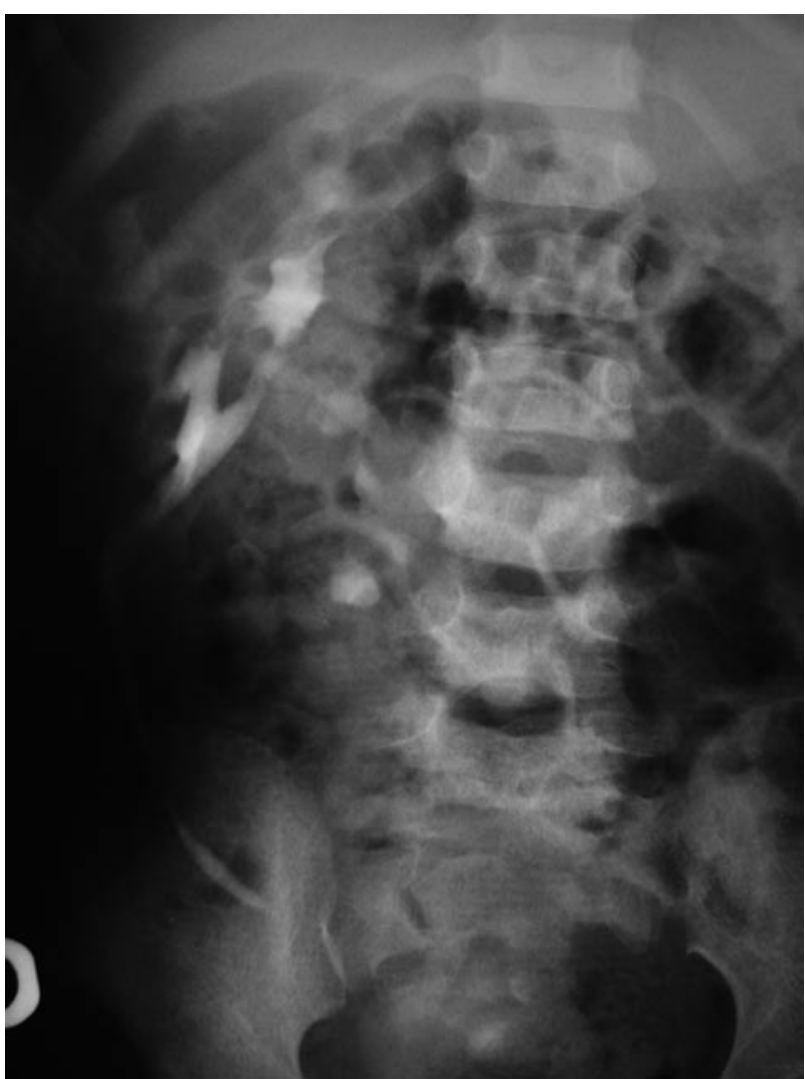

FIGURA 4. Pielografía endovenosa postoperatoria que muestra la unidad renal superior con adecuada función. 
bieron la primera nefrectomía laparoscópica de una unidad renal inferior en una ectopia renal cruzada en una niña de 12 años con un reflujo vesicoureteral grado $\mathrm{V}$ tratado con una ureteroneocistostomía Politano-Laedbetter a los tres años. La indicación quirúrgica fue una nefropatía por reflujo, cicatrices renales secundarias a pielonefritis y cólico renal. Se realizó una nefrectomía de la unidad renal inferior mediante electro cauterio bipolar, previa colocación de un catéter ureteral. En el caso que reportamos no utilizamos un catéter ureteral y la heminefrectomía se realizó mediante una técnica laparoscópica de tres puertos, con una tijera ultrasónica para sección del parénquima renal, y utilizando un variante en la posición del paciente en la mesa quirúrgica.

Nos parece necesario recalcar las ventajas en cuanto a resultados cosméticos, obtenidas al utilizar una incisión previa para extraer la pieza. Así como para la población general, el uso de la cirugía mínimamente invasiva en la población pediátrica es una realidad.

\section{CONCLUSIÓN}

El abordaje laparoscópico se presenta como una atractiva alternativa a la cirugía abierta en el manejo de esta patología. Las ventajas de la cirugía mínimamente invasiva son totalmente aplicables en este grupo etáreo, sin embargo se requieren más estudios para validar este abordaje como el tratamiento de primera línea.

\section{BIBLIOGRAFÍA y LECTURAS RECOMENDADAS ( ${ }^{*}$ lectura de interés $y^{* *}$ lectura fundamental)}

*1. Abeshouse BS, Bhisitkul I: Crossed renal ectopia with and without fusion. Urol Int, 1959; 9:63.

*2. Aguilera Tubet C, Del Valle Schaan JI, Martin Garcia B, et al: Renal Carcinoma in crossed fused renal ectopia. Actas Urol Esp, 2005; 29 (10): 993-996.

3. Cook WA, Stephens FD: Fused kidneys: morphologic study and theory of embryogenesis. Birth Defects Orig Ser, 1977; 13(5): 327-340.

4. Tsujimoto Y, Oka T, Noguchi T, et al: A case of crossed fused kidney with simple ureterocele. Nippon Hinyokika Gakkai Zasshi, 1999: 90 (12): 920-923.

5. Goodman JD, Norton KI, Carr L, et al: Crossed fused renal ectopia: sonographic diagnosis. Urol Radiol, 1986; 8 (1): 13-16.

6. Pietrow PK, Bass RA, Porter HJ 2nd. Laparoscopic hand-assisted nephrectomy for crossed fused ectopia with polycystic kidney disease. Urology, 2005; 65 (2): 388e1-e2.

**7. Stanley KE, Winfield HN, Donovan JF, et al: Laparoscopic nephrectomy in crossed fused renal ectopia. Urology 1993; 42(4): 375-378.

**8. Andersen RD, Van Savage J. Laparoscopic nephrectomy of the lower kidney for crossed fused ectopia. J Urol, 2000; 163: 1902-1903. 